\section{Identification of sequential dependencies in conversations: A Pascal program}

\author{
PORTIA E. FILE \\ Dundee Institute of Technology, Dundee, Scotland \\ and \\ JOHN TODMAN \\ University of Dundee, Dundee, Scotland
}

\begin{abstract}
A Pascal program that provides an analysis of the sequential dependencies between conversational events (e.g., speech acts) generated by two sources is described. It produces $z$ scores, chi-square values, and confidence limits for the difference between estimated unconditional probabilities of the occurrence of selected events (valid events) and estimated conditional probabilities of these events following specified target events immediately (at Lag 1) and, if required, more distantly (at Lags 2, 3, etc.). Valid events and target events may be independently selected from the set of events generated by the first source, the second source, or both. Events not selected are ignored. The user may also elect to exclude the target event from the set of valid events (an adjustment for autocorrelation). The program is especially appropriate for conversational events because it does not assume that two events from the same category can never follow one another.
\end{abstract}

The Pascal program described here is designed to analyze the sequential structure of conversational events (e.g., speech acts) generated from two distinct sources (S1 and S2) involved in a conversation. It identifies sequential dependencies between pairs of conversational events.

The analysis that the program performs is based on the method of lag-sequential analysis proposed by Sackett (1979). This type of analysis provides a measure of how likely one category of event (the target event category) is to be followed by another category of event (the valid following event category), either directly or after several intervening events (lags). When the standard analysis is chosen, the program successfully generates the same conditional probabilities as Sackett, Holm, Crowley, and Henkins's (1979) program when applied to their example data. However, our program differs from other lagsequential analysis programs (e.g., Bakeman, 1983; Sackett et al., 1979) in several ways. The most important of these, as described below, are the availability of an alternative analysis that provides an autocorrelation adjustment, a facility for separate selection of target and valid following event categories, and a change in the adjust-

Address correspondence to P. File, Department of Mathematical and Computional Sciences, Dundee Institute of Technology, Bell Street, Dundee DD1 1HG, Scotland, U.K. (e-mail: mctpf@uk.ac.dundee-tech. cc.cluster). ment for sampling method bias to adapt the statistics to conversational events.

The "autocorrelation adjusted" alternative to the standard analysis was suggested by Sackett (1987). It removes the target event category from the set of valid following event categories. Analyses with and without the adjustment can be produced by the program.

The program also allows the user to define the range of target events independently from the range of valid following events. This independence allows for several different analyses to be performed on the data. Target events contributed from S1, S2, or both can be combined in the analysis with valid following events from either S1, S2, or both. Any following events that are not defined as valid following events in a particular analysis are ignored. The independent specification of target and valid following events allows, for example, for an exploration of how S2 responds to an S1 question, with any intervening S1 speech acts ignored. An "overlapping ranges"' adjustment is required when the range for target events and the range for valid following events overlap.

The estimated probabilities and derived statistics produced by the program differ from those produced by other programs, because our data have different characteristics from those that Sackett considers and therefore require different adjustments for sampling method bias. In particular, Sackett's assumption that an event category cannot directly follow itself because of a "mutual exclusivity requirement"' (Sackett, 1979, p. 637; 1987, pp. 861$862,867)$ is incorrect for some data. Conversational events, unlike the events considered by Sackett, are not defined by a change from one event category, such as a question, to another event category, such as a statement. They are defined as subjectively meaningful events such as discrete speech acts. Thus, two events from the same event category can follow one another; for example, a question from S1 may be followed directly by another question from S1. We therefore make different adjustments for "sampling method bias," which in turn produce different estimated probabilities used to derive statistics.

The program provides both $95 \%$ and $99 \%$ confidence limits and chi-square values for each lag as well as the binomial $z$ statistic. We accept Sackett's (1987, p. 869) conclusion that this binomial $z$ statistic is more conservative and, in view of the large numbers of statistical decisions being made, preferable to the normal-distribution $z$ statistic proposed by Allison and Liker (1982).

The program was used by Todman, Elder, Alm, and File (in press) to analyze sequential dependencies in computer-aided conversations to compare the structure of these conversations with the structure of conversations between unaided participants. Specifically, the analysis was used to examine similarities in sequential dependencies for categories of conversational events in the two types of conversations. 


\section{The Data}

Conversational events (e.g., speech acts) by one participant in a conversation (S1) are coded as integers, with each integer representing a category of interest to the researcher (e.g., a question may be coded as 11 , an answer as 12 , an observation as 13 , an agreement as 14 , etc.). Conversational events by the other participant in the conversation (S2) are similarly coded. The categories for S2 may be the same as or different from the categories for $\mathrm{S} 1$. For example, Categories 21,22 , and 23 for $\mathrm{S} 2$ may be a question, an answer, or an observation corresponding to Categories 11,12 , and 13 for $S 1$, or they could represent entirely different kinds of events.

The sequence of integers representing the sequence of conversational events for a particular interaction between $\mathrm{S} 1$ and $\mathrm{S} 2$ is the subject of the analysis.

For example, a conversation with the following sequence of speech acts: question(S1), answer(S2), observation(S2), question(S1), answer(S2), agreement(S1), observation(S2), question(S1), answer(S2), agreement(S1) might be coded, as described above, as 1122231122 1423112214 .

\section{Analysis}

The program calculates the observed frequency of different sequences of conversational events and the appropriate inferential statistics for these frequencies. In particular, the number of times each member of one specified set of conversational event categories (termed target events) is followed by a particular member of another specified set of conversational event categories (termed valid events) is counted. The first valid event that follows a target event is tallied as a Lag 1 occurrence. If required, the second, third, and subsequent occurrences of a valid event after a target event can also be tallied as Lag 2, Lag 3, and so on. On the basis of these observed frequencies, estimates of the conditional probability of each valid event following a target event at each lag are calculated. Further, the overall frequency of each valid event is used to calculate the estimated unconditional probability of the occurrence of that valid event. The difference between the conditional probability of each valid event following the target at each lag and the unconditional probability of that valid event is obtained. Then $z$ scores and overall chi-square values for these differences, as well as $95 \%$ and $99 \%$ confidence limits, are calculated.

More specifically, the program finds each occurrence of an item in the set of initial events (target events). Each time a target is found, the program identifies which of a set of following events of interest (valid events) occurs first after the target (Lag 1) and, if required, which valid event occurs next (Lag 2), and so on for additional lags. These identified items are then tallied. When completed, the program produces the total number of occurrences of each valid event that follows occurrences of each target event at each LAG. Events not specified are ignored.

The user can control several aspects of the information collected. The counts of occurrences of valid events are carried out for each lag from Lag 1 up to a maximum lag value (specified by the user at runtime). The range of the set of target events and the set of valid events to be searched for in locations following each occurrence of a target event are also specified at runtime. Note that these two ranges may overlap fully, partially, or not at all, and a further choice by the user is required to specify the desired treatment for overlapping ranges.

For each valid event, the program obtains its estimated conditional lag probabilities (i.e., probabilities of following from one to maximum lag steps after a particular target event) and its unconditional probability. The conditional lag probabilities are estimated from observed frequencies of each valid event at each lag. The estimated unconditional probability for each valid event is determined by dividing the overall frequency of that valid event by the total number of valid events available to follow the target event.

The total number of available valid events is calculated by adjusting the total number of valid events to take account of reductions in the number that are available to follow the target event. The reductions are due to (1) a target being within the range of valid events to be searched, and (2) the special case of (1), in which a search is conducted for a valid event from the same category as the current target event. The "overlapping range" adjustment required for case (1) reduces the total number of available valid events by one, because the current target event itself is not available. The "sampling method bias" adjustment required for case (2) requires reduction of available valid events within the target category by one, again because the current target event, though a member of the target category, is not available.

With overlapping ranges for target events and valid events, there is no restriction on a target's being followed by itself (as a valid event) at Lag 1 (e.g., an S1 "observation" valid event can follow immediately after another S1 "observation" target event). Event categories that follow themselves in this way are referred to as autolags to distinguish them from event categories that follow a different event category (referred to as crosslags). The user chooses at runtime whether or not to remove, from the crosslag sequential analysis, the effects of autocorrelation (i.e., autolag dependency of an event category on a previous occurrence of itself), as suggested by Sackett (1987). The null hypothesis that conditional lag probabilities do not differ from estimated unconditional probabilities can be tested by $z$ and chi-square statistics. Confidence intervals of $99 \%$ and $95 \%$ are also provided.

\section{Program Details}

The program is written in standard Pascal. The constraints of standard Pascal and of some operating systems have necessitated a degree of inelegance in the program interfaces, but the benefits of portability were judged to outweigh this awkwardness.

The sequence of events to be analyzed is represented as a sequence of integers in a data file with one integer per line ending with the end of data value. (Initially, "end- 
ofdata" is set to 999 but it can be changed.) The maximum number of data values is set to the value of " $\max$ events" (initially 2,000 , but this can be changed). The data file must be in the current directory and have the name "data.dat."

At runtime, the program requests (1) the specified maximum lag, which should not exceed the value of "toplag" (initially 10, but can be changed); (2) the codes for the first and last in the range of event categories that are to be used as targets; (3) a switch (the program variable, "valideventswitch") to determine whether S1 (i.e., event codes from the "maxstartevent" to the "midevent," initially 11-20), S2 (i.e., event codes from the midevent to the maxendevent, initially $20-29$ ), or both sources ( $\max -$ startevent to maxendevent, initially 11-29) are to be considered valid events (i.e., to be searched for at the specified lags); and (4) a switch (the program variable, "autoadjust") to select the standard analysis (based on both autolags and crosslags) or analysis with the effects of autocorrelation removed.

Results are output to a file named "results.dat" in the current directory. The results consist of a set of tables for each target event for each lag, giving observed and expected frequencies, estimated conditional and unconditional probabilities, $z$ scores, $95 \%$ and $99 \%$ confidence limits, and chi-square values.

\section{A Sample Analysis}

The sample conversation described above would be coded as 11222311221423112214999 , where the 999 indicates the end of the data. These data should be in a file named "data.dat," with one integer per line. This sequence is essentially the same as the example used by Sackett et al. (1979), which is A B D A B C D A B C.

Appendix A contains the required dialogue for a standard analysis of this conversation without an adjustment for autocorrelation, with a maximum lag of 1 (Lag 1 only analyzed), with Category 11 only as the target event, and with all events used as valid events (Categories 11-29).

The results for an analysis are stored in the file named "results.dat," and those for the above example are given in Appendix B. If subsequent analyses are to be performed, the file should be renamed or printed first.

For each analysis, the results file contains a brief description of the options selected. This is followed by a list of the valid event categories (which serves as column headings for all subsequent tables), a table giving the frequency of each valid event, and the total number of valid events ( $N$ total).

Then, for each target, tables of counts and derived statistics (from Sackett, 1979) are presented under a number of headings, which are reproduced below with explanations of what appears under them. For each target, the first table described below is given once, while the others are repeated for each lag.

Estimated Unconditional Probability of Valid Events gives the frequency of each valid event divided by the total number of valid events, corrected for "overlapping ranges," if appropriate. This correction reduces the total number of valid events by one when the target and valid event ranges overlap. In this example, the target range consists only of Category 11 but this overlaps with the valid event range, which is from Categories 11-29. The total number of valid events that can follow a target in this analysis is therefore 9 rather than 10 because the current target event cannot itself be a valid event.

Observed Frequencies of Valid Events gives the frequency of each valid event observed at that lag for the target, corrected for "sampling bias" if the valid event is the target event. This correction reduces the frequency of valid events by one when the target is in the same category as the valid event. In this example, the frequency of Category 11 is reduced from three to two when 11 is the target, because only two Category 11 events can follow the target Category 11 event.

Estimated Conditional Probabilities of Valid Events gives the frequency of each valid event following the target at a given lag, divided by the total number of valid events occurring at that lag. This total is usually equal to the number of targets, but may be lower if targets occur near the end of the data so no events occur at some, particularly longer, lags.

Expected Frequency of Valid Events gives the estimated unconditional probability of each valid event multiplied by the total number of valid events occurring at that lag.

$Z$ gives the $z$ score for the difference between the estimated conditional probability for each valid event at that lag and the estimated unconditional probability for that valid event.

Confidence Intervals (99\% and $95 \%$ ) give confidence limits for the expected probabilities.

Chi Square gives the chi-square values for estimated probabilities. It is followed by a sum of chi-square, which is the sum of the chi-squares that are significant at the .05 level (two-tailed).

For the corresponding analysis of the same data with an adjustment for autocorrelation, the user should respond with " 1 " to the dialogue query "run type-0 for normal run, 1 for autocorrelation adjustment."

The results for this second analysis on the sample data with the autocorrelation adjustment are given in Appen$\operatorname{dix} C$. They differ from the analysis without the autocorrelation adjustment because the frequency of the valid events in the target category is set to zero and the total number of valid events is adjusted accordingly before any analyses are carried out.

\section{Required Resources}

Two different versions of the program are available. For maximum portability, a very general version is supplied as ASCII source code, which should run on any computer platform with either an ISO standard or ANSI standard Pascal compiler. It has been tested with the sample data file on a VAX/VMS system in which the files used about $40 \mathrm{~K}$ disk storage and about $1.5 \mathrm{MB}$ memory at runtime. For this operating system, the data and results file 
names must be "data.dat" and "results.dat," respectively. The ".dat" extension for the data and results file names may not be appropriate for other operating systems. In all cases, the appropriate default extensions for the operating system should be used.

A second version for IBM-compatible computers (AT and upward) is supplied in executable form. This version allows the user to input the name of the data file and the name of the results file. It requires less than 20K disk space.

\section{Program Availability}

Copies of the program may be obtained from the first author either by sending an electronic mail request to mctpf@uk.ac.dundee-tech.cc.cluster, or by sending a formatted 3.5-in. or 5.25-in. high-density floppy disk to P. File, Department of Mathematical and Computer Sciences, Dundee Institute of Technology, Bell Street, Dundee, DD1 1HG, Scotland, U.K.

\section{REFERENCES}

Allison, P. D., \& LIKER, J. K. (1982). Analyzing sequential categorical data on dyadic interaction: A comment on Gottman. Psychological Bulletin, 91, 393-403.

BAKEMAN, R. (1983). Computing lag sequential statistics: The ELAG program. Behavior Research Methods \& Instrumentation, 15, 530-535.

SACKETT, G. P. (1979). The lag sequential analysis of contingency and cyclicity in behavioral interaction research. In J. D. Osofsky (Ed.), Handbook of infant development (pp. 623-649). New York: Wiley.

SACKETT, G. P. (1987). Analysis of sequential social interaction data: Some issues, recent developments, and a causal inference model. In
J. D. Osofsky, (Ed.), Handbook of infant development (pp. 855-878). New York: Wiley.

Sackett, G. P., Holm, R., Crowley, C., \& Henkins, A. (1979). Computer technology: A Fortran program for lag sequential analysis of contingency and cyclicity in behavioral interaction data. Behavior Research Methods \& Instrumentation, 11, 366-378.

Todman, J., Elder, L., Alm, N., \& File, P. (in press). Sequential dependencies in computer-aided conversation. Journal of Pragmatics.

\section{APPENDIX A \\ Example Computer Dialogue for the Standard Analysis}

The data file should be in the current directory and should have the file name "data.dat." The results will be produced in the file "results.dat."

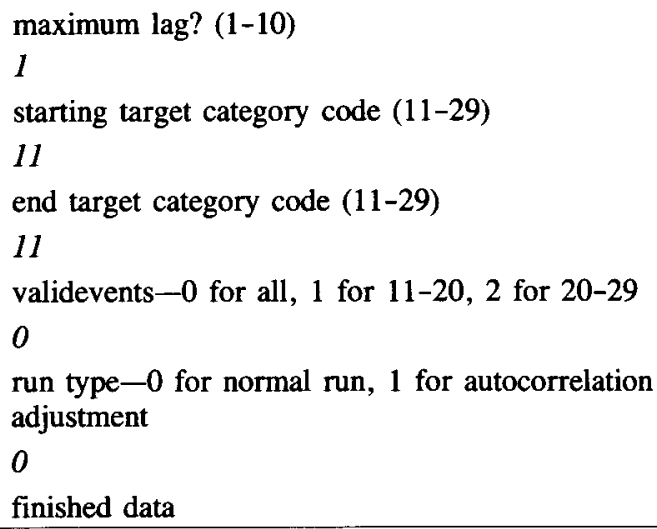

\section{APPENDIX B}

Standard Analysis Results

Lags $=1-1$

Target Range $=11-11$

Valid Following Event Range = 11-29

Valid Event Categories

$\begin{array}{lllllllllllllllllll}11 & 12 & 13 & 14 & 15 & 16 & 17 & 18 & 19 & 20 & 21 & 22 & 23 & 24 & 25 & 26 & 27 & 28 & 29\end{array}$

Frequency of Each Valid Event

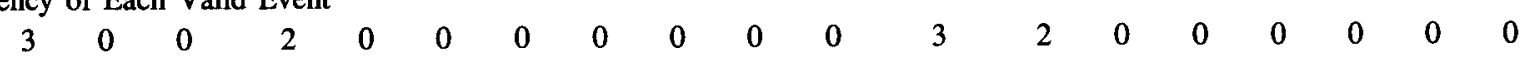

$N$ total $=10$

Target $=11$

Estimated Unconditional Probabilities of Valid Events

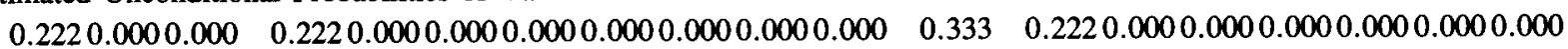

Target $=11 \mathrm{LAG}=1$

Observed Frequencies of Valid Events

$$
\begin{array}{llllllllllllllllllll}
0 & 0 & 0 & 0 & 0 & 0 & 0 & 0 & 0 & 0 & 0 & 3 & 0 & 0 & 0 & 0 & 0 & 0 & 0
\end{array}
$$

Estimated Conditional Probabilities of Valid Events

$$
\begin{array}{llllll}
0.0000 .0000 .000 & 0.0000 .0000 .0000 .0000 .0000 .000 & 0.0000 .000 & 1.000 & 0.0000 .0000 .0000 .0000 .0000 .0000 .000
\end{array}
$$

Expected Frequency of Valid Events

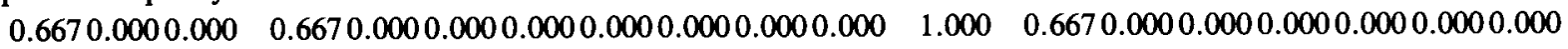

$Z$ 
Confidence Interval $99+$ $\begin{array}{lllll}0.8370 .000 & 0.000 & 0.8370 .0000 .0000 .0000 .0000 .0000 .0000 .000 & 1.030 & 0.8370 .0000 .0000 .0000 .0000 .0000 .000\end{array}$

Confidence Interval $99-$

$-0.3920 .0000 .000-0.3920 .0000 .0000 .0000 .0000 .0000 .0000 .000-0.363-0.3920 .0000 .0000 .0000 .0000 .0000 .000$

Confidence Interval $95+$

$\begin{array}{lllll}0.6930 .0000 .000 & 0.6930 .0000 .0000 .0000 .0000 .0000 .0000 .000 & 0.867 & 0.6930 .0000 .0000 .0000 .0000 .0000 .000\end{array}$

Confidence Interval $95-$

$-0.2480 .0000 .000-0.2480 .0000 .0000 .0000 .0000 .0000 .0000 .000-0.200-0.2480 .0000 .0000 .0000 .0000 .0000 .000$

Chi Square

$\begin{array}{lllll}0.6670 .0000 .000 & 0.6670 .0000 .0000 .0000 .0000 .0000 .0000 .000 & 4.000 & 0.6670 .0000 .0000 .0000 .0000 .0000 .000\end{array}$

Note-Sum of chi squares $=0.000$. Total frequencies of valid events at each lag $=3$.

\section{APPENDIX C}

Autocorrelation Adjusted Analysis Results

Lags $=1-1$

Target Range $=11-11$

Valid Following Event Range $=11-29$

Autocorrelation Adjustment

Valid Event Categories

$\begin{array}{llllllllllllllllllllll}11 & 12 & 13 & 14 & 15 & 16 & 17 & 18 & 19 & 20 & 21 & 22 & 23 & 24 & 25 & 26 & 27 & 28 & 29\end{array}$

Frequency of Each Valid Event

$\begin{array}{ccccccccccccccccccccccc}3 & 0 & 0 & 2 & 0 & 0 & 0 & 0 & 0 & 0 & 0 & 3 & 2 & 0 & 0 & 0 & 0 & 0 & 0\end{array}$

$N$ total $=10$

Target $=11$

Estimated Unconditional Probabilities of Valid Events

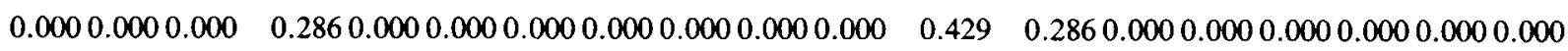

Target $=11$, Lag $=1$

Observed Frequencies of Valid Events

$\begin{array}{lllllllllllllllllllllll}0 & 0 & 0 & 0 & 0 & 0 & 0 & 0 & 0 & 0 & 0 & 3 & 0 & 0 & 0 & 0 & 0 & 0 & 0\end{array}$

Estimated Conditional Probabilities of Valid Events

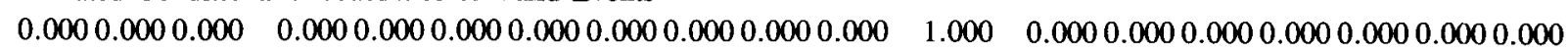

Expected Frequency of Valid Events

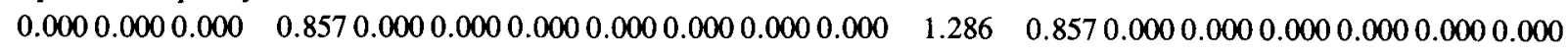

$Z$

$0.0000 .0000 .000-1.0950 .0000 .0000 .0000 .0000 .0000 .0000 .0002 .000-1.0950 .0000 .0000 .0000 .0000 .0000 .000$

Confidence Interval $99+$

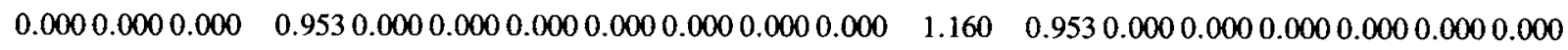

Confidence Interval $99-$

$0.0000 .0000 .000-0.3820 .0000 .0000 .0000 .0000 .0000 .0000 .000-0.303-0.3820 .0000 .0000 .0000 .0000 .0000 .000$

Confidence Interval $95+$

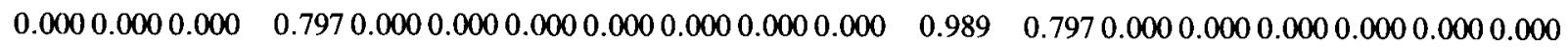

Confidence Interval $95-$

$0.0000 .0000 .000-0.2250 .0000 .0000 .0000 .0000 .0000 .0000 .000-0.131-0.2250 .0000 .0000 .0000 .0000 .0000 .000$

Chi Square

$\begin{array}{lllll}0.0000 .0000 .000 & 0.8570 .0000 .000 & 0.0000 .0000 .0000 .0000 .000 & 2.286 & 0.8570 .0000 .0000 .0000 .0000 .0000 .000\end{array}$

Note-Sum of chi squares $=0.000$. Total frequencies of valid events at each lag $=3$. 